\title{
Effect of Peg-6000 Induced Drought on Physiological Indices and Correlation of Seedling Stage Traits in Maize (Zea mays L.) Hybrids
}

\author{
R. Nirmal Raj*, C.P. Renuka Devi and J. Gokulakrishnan \\ Department of Genetics and Plant Breeding, Annamalai University, India \\ *Corresponding author
}

\section{A B S T R A C T}

\begin{tabular}{|l|}
\hline Ke y w o r d s \\
Osmotic stress, \\
PEG-6000, \\
$\begin{array}{l}\text { Drought, } \\
\text { Promptness index, } \\
\text { Association analysis }\end{array}$ \\
\hline Article Info \\
\hline $\begin{array}{l}\text { Accepted: } \\
\text { 12 December } 2018 \\
\text { Available Online: } \\
\text { 10 January } 2019\end{array}$ \\
\hline
\end{tabular}

\section{Introduction}

Maize is an interesting crop in terms of its wide area of cultivation and hybrid yield potential however; various abiotic stresses limit the magnitude of yield. Water stress due to drought is one such stress that plays a significant role in crop growth and development (Hartman et al., 2005).

The never ending demand for maize crop and growing monsoon failure has tilted the objectives of plant breeding towards the identification of hybrids tolerant to water stress (Avramova et al., 2015). The screening for water stress is facilitated by a high molecular weight chemical called PEG-6000 and this induces varying osmotic pressure at different concentrations (Hardegree and Emmerich, 1990).

It is a better agent than mannitol as the latter could induce certain toxic effects to the growth of a plant (Hohl and Schopfer, 1991) and is an able laboratory screening method.

Hence, an attempt was made to understand the effects PEG-6000 on seedling vigour indices and the association between traits under drought. 


\section{Materials and Methods}

The experiment was conducted at Abiotic stress laboratory, Annamalai University using twenty one maize hybrids and the design laid out was completely randomized design. Seeds of homogenous size were selected and placed in petridishes with germination papers. Ten seeds of each hybrid were placed in petridishes which was treated with corresponding PEG treatments diluted in distilled water (control, 10\% and 20\%). The osmotic pressure exerted by these concentrations was estimated using Michel's formulae and the estimates were 0 bar, -3 bars and -6 bars respectively at $32^{\circ} \mathrm{C}$.

$\Psi \mathrm{s}=-\left(1.18 \times 10^{-2}\right) \mathrm{C}-\left(1.18 \times 10^{-4}\right) \mathrm{C}_{2}+$ $\left(2.67 \times 10^{-4}\right) \mathrm{CT}+\left(8.39 \times 10^{-7}\right) \mathrm{C}_{2} \mathrm{~T}$

Where, $\Psi \mathrm{s}=$ Osmotic potential (bar)

$\mathrm{C}=$ Concentration $\left(\mathrm{g} \mathrm{L}^{-1}\right.$ PEG-6000 in water)

$\mathrm{T}=$ Temperature $\left({ }^{\circ} \mathrm{C}\right)$

Seven physiological traits were recorded viz., germination percentage (\%), shoot length $(\mathrm{cm})$, root length $(\mathrm{cm})$, seminal root length $(\mathrm{cm})$, fresh weight $(\mathrm{g})$, dry weight $(\mathrm{g})$ and seed vigour index. The duration of experiment spanned for seven days and the data recorded were subjected to mean performance analysis and character correlation assessment.

\section{Results and Discussion}

Combined analysis of variance was carried out which showed significant variation among hybrids and significant effect of PEG-6000 treatments on all the seven morphological traits (Table 1), hence an assessment of the effect of PEG solution on the observed indices were possible (Partheeban et al., 2017).

Main effects of drought stress levels on the physiological indices were analyzed by comparing the per se performance which revealed high limiting effects on all the characters (Table 2). The most limited traits due to water stress were germination percentage followed by seedling vigour index. The rapid reduction in germination percentage is due to inefficient cell division and plant growth metabolism (Ayaz et al., 2001). Root length increased under osmotic potential of -3 bars but under severe osmotic pressure the length decreased, emphasizing the role of vigorous root growth at seedling stage which would result in better root structure at maturity (Nejad, 2011).

Table.1 Analysis of variance of observed traits in maize hybrids under drought stress

\begin{tabular}{|c|c|c|c|c|c|c|c|c|}
\hline Source & df & $\begin{array}{c}\text { EP } \\
(\mathbf{\%})\end{array}$ & $\begin{array}{c}\text { SL } \\
(\mathbf{c m})\end{array}$ & $\begin{array}{c}\text { RL } \\
(\mathbf{c m})\end{array}$ & $\begin{array}{c}\text { SRL } \\
(\mathbf{c m})\end{array}$ & $\begin{array}{c}\text { FW } \\
(\mathbf{g})\end{array}$ & $\begin{array}{c}\text { DW } \\
(\mathbf{g})\end{array}$ & $\begin{array}{c}\text { SV I } \\
(\mathbf{\%})\end{array}$ \\
\hline Genotype & 20 & $462.14^{* *}$ & $1.43^{* *}$ & $6.32^{* *}$ & $4.11^{* *}$ & $0.03^{* *}$ & $0.01^{* *}$ & $90366.88^{* *}$ \\
\hline Treatment & 2 & $36144.05^{* *}$ & $60.29^{* *}$ & $59.84^{* *}$ & $82.43^{* *}$ & $0.42^{* *}$ & $0.08^{* *}$ & $2552009.00^{* *}$ \\
\hline G $\times \mathrm{T}$ & 40 & $120.30^{* *}$ & $0.41^{* *}$ & $1.31^{* *}$ & $0.79^{* *}$ & $0.02^{* *}$ & $0.01^{* *}$ & $18480.88^{* *}$ \\
\hline Error & 60 & 5.99 & 0.00 & 0.00 & 0.00 & 0.00 & 0.00 & 376.51 \\
\hline
\end{tabular}

*: Significant at $5 \%$ level; $\quad * *$ : Significant at $1 \%$ level

EP- Emergence percentage SL- Shoot length, SLSI- Shoot length stress index, RL- Root length, SRL- Seminal root length, FW- Fresh weight, DW- Dry weight, SV I- Seed vigour I. 
Table.2 Mean comparison of main effects of drought stress levels

\begin{tabular}{|c|c|c|c|c|c|c|c|}
\hline $\begin{array}{c}\text { Drought } \\
\text { Stress }\end{array}$ & $\begin{array}{c}\text { EP } \\
(\%)\end{array}$ & $\begin{array}{c}\text { SL } \\
(\mathbf{c m})\end{array}$ & $\begin{array}{c}\text { RL } \\
(\mathbf{c m})\end{array}$ & $\begin{array}{c}\text { SRL } \\
(\mathbf{c m})\end{array}$ & $\begin{array}{c}\text { FW } \\
(\mathbf{g m})\end{array}$ & $\begin{array}{c}\text { DW } \\
(\mathbf{g m})\end{array}$ & $\begin{array}{c}\text { SV I } \\
(\mathbf{\%})\end{array}$ \\
\hline Control & 96.67 & 3.58 & 4.26 & 4.39 & 0.48 & 0.24 & 758.52 \\
\hline $\begin{array}{c}\mathrm{T}_{1} \\
(10 \%)\end{array}$ & 46.90 & 1.66 & 5.19 & 3.71 & 0.43 & 0.22 & 353.97 \\
\hline $\begin{array}{c}\mathrm{T}_{2} \\
(20 \%)\end{array}$ & 14.29 & 0.20 & 1.91 & 0.67 & 0.22 & 0.12 & 64.49 \\
\hline
\end{tabular}

Table.3 Genotypic and phenotypic correlation among physiological indices (Control)

\begin{tabular}{|c|c|c|c|c|c|c|c|c|}
\hline \multicolumn{2}{|c|}{ Characters } & EP & SL & RL & SRL & FW & DW & SV I \\
\hline \multirow[t]{2}{*}{ EP } & G & 1.000 & -0.107 & 0.204 & $0.456 *$ & -0.131 & -0.193 & 0.244 \\
\hline & $\mathrm{P}$ & 1.000 & -0.107 & 0.204 & $0.454 *$ & -0.131 & -0.192 & 0.244 \\
\hline \multirow[t]{2}{*}{ SL } & G & & 1.000 & $0.508 *$ & 0.155 & 0.037 & -0.143 & $0.790 * *$ \\
\hline & $\mathrm{P}$ & & 1.000 & $0.508 *$ & 0.154 & 0.037 & -0.142 & $0.790 * *$ \\
\hline \multirow[t]{2}{*}{$\mathbf{R L}$} & G & & & 1.000 & $0.487 *$ & 0.240 & 0.229 & $0.910 * *$ \\
\hline & $\mathrm{P}$ & & & 1.000 & $0.485 *$ & 0.240 & 0.228 & $0.910 * *$ \\
\hline \multirow[t]{2}{*}{ SRL } & G & & & & 1.000 & -0.077 & -0.137 & $0.475^{*}$ \\
\hline & $\mathrm{P}$ & & & & 1.000 & -0.074 & -0.131 & 0.473* \\
\hline \multirow[t]{2}{*}{ FW } & G & & & & & 1.000 & $0.877 * *$ & 0.146 \\
\hline & $\mathrm{P}$ & & & & & 1.000 & $0.877 * *$ & 0.146 \\
\hline \multirow[t]{2}{*}{ DW } & G & & & & & & 1.000 & 0.032 \\
\hline & $\mathrm{P}$ & & & & & & 1.000 & 0.033 \\
\hline \multirow[t]{2}{*}{ SV I } & G & & & & & & & 1.000 \\
\hline & $\mathrm{P}$ & & & & & & & 1.000 \\
\hline
\end{tabular}

*: Significant at 5\% level; $\quad$ **: Significant at $1 \%$ level

Table.4 Genotypic and phenotypic correlation among physiological indices under drought

\begin{tabular}{|c|c|c|c|c|c|c|c|c|}
\hline \multicolumn{2}{|c|}{ Characters } & EP & SL & RL & SRL & FW & DW & SV I \\
\hline \multirow[t]{2}{*}{ EP } & G & 1.000 & $0.981 * *$ & $0.888 * *$ & $0.927 * *$ & $0.720 * *$ & $0.751 * *$ & 0.956 ** \\
\hline & $\mathrm{P}$ & 1.000 & 0.959 ** & $0.869 * *$ & $0.907 * *$ & $0709 * *$ & $0.743 * *$ & $0.955 * *$ \\
\hline \multirow[t]{2}{*}{ SL } & G & & 1.000 & $0.912 * *$ & $0.937 * *$ & $0.777 * *$ & $0.788 * *$ & $0.917 * *$ \\
\hline & $P$ & & 1.000 & $0.910 * *$ & $0.935 * *$ & $0.775 * *$ & $0.785 * *$ & $0.905^{* * *}$ \\
\hline \multirow[t]{2}{*}{ RL } & G & & & 1.000 & $0.903 * *$ & $0.900 * *$ & $0.903 * *$ & 0.818** \\
\hline & $\mathrm{P}$ & & & 1.000 & $0.902 * *$ & $0.900 * *$ & $0.901 * *$ & $\mathbf{0 . 8 0 8}^{* *}$ \\
\hline \multirow[t]{2}{*}{ SRL } & G & & & & 1.000 & $0.714^{* * *}$ & $0.708 * *$ & $0.881 * *$ \\
\hline & $\mathrm{P}$ & & & & 1.000 & $0.714 * *$ & $0.707 * *$ & 0.870 *** \\
\hline \multirow[t]{2}{*}{ FW } & G & & & & & 1.000 & $0.972 * *$ & $0.563 * *$ \\
\hline & $\mathrm{P}$ & & & & & 1.000 & $0.971 * *$ & 0.560 ** \\
\hline \multirow[t]{2}{*}{ DW } & G & & & & & & 1.000 & $0.607 * *$ \\
\hline & $\mathrm{P}$ & & & & & & 1.000 & $0.605 * *$ \\
\hline \multirow[t]{2}{*}{ SV I } & G & & & & & & & 1.000 \\
\hline & $\mathrm{P}$ & & & & & & & 1.000 \\
\hline
\end{tabular}


Correlation between traits was analyzed under both normal and severe drought conditions (Table 3 and 4). Under normal condition both shoot and root length showed high positive association towards seed vigour index, whereas under severe osmotic stress all the six characters were positively correlated to seed vigour index of which germination percentage was of highest magnitude followed by shoot length and seminal root length. Similar findings were reported by Khan et al., (2004) in maize hybrids.

Considering the above findings, the physiological traits viz., germination percentage, shoot length and root length are able factors aiding indirect selection for drought tolerant hybrids. Germination percentage is the candidate trait that determines seed vigour index under stress conditions.

\section{References}

Avramova, V., Knapen, D., Taleisnik, E. and Beemster, G.T.S. 2015. Drought induces distinct growth response, protection, and recovery mechanisms in the maize leaf growth zone. Plant. Physiol. 169: 1382-1396.

Ayaz, F. A., Kadioglu, A. and Urgut, R. T. 2001. Water stress effects on the content of low molecular weight carbohydrates and phenolic acids in
Cienanthe setosa. Canadian J. Plant Sci. 80: 373-378.

Hardegree, S.P. and Emmerich, W. E. 1990. Effect of polyethylene glycol exclusion on the water potential of solutionsaturated filter paper. Plant Physiol. 92: 462-466.

Hartmann, T., College, M. and Lumsden, P. 2005. Responses of different varieties of Lolium perenne to salinity. Annual Conference of the Society for Experimental Biology, Lancashire.

Hohl, M. and Schopfer, P. 1991. Water relations of growing Maize coleoptiles comparison between Mannitol and Polyethylene glycol 6000 as external osmotica for adjusting turgor pressure. Plant Physiology. 95(3): 716-722.

Khan, A.A., Sajjad, A.R. and McNeilly, T. 2004. Assessment of salinity tolerance based upon seedling root growth response functions in maize (Zea mays L.). Euphytica. 131: 81-89.

Nejad, T. S. 2011. Effect of drought stress on shoot/root ratio. World Acad. Sci. Eng. Technol. pp. 598-600.

Partheeban, C., Chandrasekhar, C.N., Jeyakumar, P., Ravikesavan, R. and Gnanam, R. 2017. Effect of PEG induced drought stress on seed germination and seedling characters of maize (Zea mays L.) genotypes. Int. J. Curr. Microbiol. App. Sci. p. 10951104.

\section{How to cite this article:}

Nirmal Raj, R., C.P. Renuka Devi and Gokulakrishnan, J. 2019. Effect of Peg-6000 Induced Drought on Physiological Indices and Correlation of Seedling Stage Traits in Maize (Zea mays L.) Hybrids. Int.J.Curr.Microbiol.App.Sci. 8(01): 1642-1645.

doi: https://doi.org/10.20546/ijcmas.2019.801.172 